\title{
Nutritional Knowledge and Behavior of Professional Athletes in Khorramabad
}

\author{
Mahnaz Mardani ${ }^{1}$, Behzad Bazgir ${ }^{2}$, Akram Beigi Bazgir ${ }^{3}$, Hossein Mansurnezhad ${ }^{4}$ and Maryam Rezapour ${ }^{5}$ \\ 1. Nutritional Health Research Center, Department of Health \& Nutrition, Lorestan University of Medical Sciences, Khorramabad, \\ Iran
}

2. Exercise Physiology Research Center, Baqiyatallah University of Medical Science, Tehran, Iran

3. Department of Health \& Nutrition, Lorestan University of Medical Science, Iran

4. Department of Physical Education and Sport Science, Faculty of literature and Humanities, Lorestan University, Khorramabad, Iran

5. Department of Pharmacy, Arvand Branch of Ahvaz Jundishapur University of Medical Sciences, Ahvaz, Iran

\begin{abstract}
Today it is proved that the lack of adequate nutritional knowledge leads to problems such as obesity, overweight and increased cardiovascular diseases. However, many obstacles such as poor financial support, lack of time and poor nutritional knowledge can influence achievement of optimal nutrition which is proportional to the level of the athlete's physical activity. The purpose of this study was to determine the level of nutritional knowledge and dietary behavior of athletes in Khorramabad in 2013. Professional athletes of Khorramabad, Iran, with more than one year of regular exercise and at least one course participating in national championships competitions were studied in this cross-sectional, descriptive-analytic study. The samples included 247 athletes aged 17-29 years. Their nutritional knowledge and behavior were measured by the nutritional knowledge and behavior inventory. Results: The results showed a significant difference in nutritional knowledge of athletes by gender and educational level; female athletes have higher nutritional knowledge than male athletes. A gender-dependent difference was found in nutritional behavior; despite their higher nutritional knowledge, female athletes have weaker nutritional behavior than male athletes. However, no significant correlation was found between BMI and nutritional knowledge and behavior of the athletes. Moreover, findings showed that nutritional knowledge and behavior could be significantly different in various sports.
\end{abstract}

Key words: Sports, nutritional knowledge, nutritional behavior, Khorramabad.

\section{Introduction}

The lifestyle changes associated with technological advances and industrial life consequentially changes the society's nutrition, their type of food choice and mealtime. Moreover, organic food has been replaced by industrial products, which, along with the tendency to fast food restaurants, has considerably changed the health and the healthy diet of people. Research has shown that the lack of adequate nutritional knowledge could lead to problems such as obesity, overweight and increased cardiovascular diseases [1]. The main aims of participating in physical activities and sports

Corresponding author: Mahnaz Mardani, associate professor, research field: nutrition. are to enhance the level of health, control weight and improve athletic performance. Sport and physical activity has beneficial effects on improved human body functions, including cardiovascular, respiratory, neuromuscular and gastrointestinal systems. Whereas, physical activity without healthy nutrition can cause weakness or dysfunction of various organs. However, many obstacles such as weak financial support, insufficient time and poor nutritional knowledge can affect an athlete's optimal nutrition which is proportional to physical activity. Studies have shown that athletes still do not have enough knowledge on healthy nutrition; nutritional information is delivered through various sources such as coaches, teammates, 
nutrition consultants, fitness trainers, parents, distributors of sports nutrition supplements, media and other athletes [2]. In Iran, teams and even universities lack the sports science and nutritional services to provide the best source of nutritional information for athletes. Moreover, a relationship has not been well established between science and sports. In Khorramabad, sports teams lack nutrition consultants and athletes need to refer to other sources to resolve their nutritional problems. Some of these sources do not provide proper nutritional information and sometimes spread unreliable information which is only propaganda. This could adversely influence the nutrition and athletic performance of athletes [3]. Studies have shown that optimal nutrition can reduce fatigue and allow athletes to train and compete at longer durations or recover faster between training sessions [4]. Nutritional behavior of Iranians has changed in the past decade [5]. It is essential to take into account the nutritional considerations in proper diet of athletes, particularly young and female athletes, due to their higher metabolic and energy requirements. Wardle et al. [6] noted that nutritional knowledge can have an important role in a variety of food choices, and nutritional knowledge is a relatively effective intervening factor in socio-demographic differences in consumed foods, particularly fruits and vegetables. Nutrition plays a very important role in achieving high levels of sports performance. Nutritional status is directly related to levels of body composition [7]. Fitness and exercise are highly dependent on the nutritional status of participants in sports. Nutrition is an important supplement for any type of physical fitness program. It is well known that appropriate nutrition can improve athletic performance. On the other hand, recent studies show that nutritional knowledge and attitude can affect eating habits [1]; thus, good nutritional knowledge, habits and attitudes alongside improved athletes nutrition could be practical approaches in enhancing athletic performance.

In a cross-sectional study, Jessri et al. [3] evaluated nutritional knowledge of 66 basketball player students and 141 soccer player students. They found that females acquired higher scores in nutritional knowledge than males; moreover, students of medical sciences and those who received nutritional information from reliable sources obtained a higher score in nutritional knowledge than students in other universities. Nearly $90 \%$ of athletes considered coaches as the main source of nutritional knowledge. Finally, authors concluded that athletes did not have enough nutritional knowledge [3].

By evaluating nutritional knowledge of 250 collegiate and non-collegiate (130 male, 120 female) athletes, Arazi and Hosseini [8] found that nutritional knowledge of non-collegiate athletes is lower than collegiate athletes.

No study has been conducted so far on nutritional knowledge and behavior of athletes in Khorramabad. Since these athletes are representative of Khorramabad in national competitions, they require a proper level of nutritional knowledge and behavior for a successful athletic performance. Therefore, this study was carried out to determine the level of nutritional knowledge and dietary behavior of athletes in Khorramabad in 2013.

\section{Materials and Methods}

In this descriptive-analytically cross-sectional study, all professional athletes of Khorramabad County with more than one year of regular exercise and at least one course of participation in national championship were allocated as the study population. Athletes of active fields including volleyball, freestyle and Greco-Roman wrestling, track and field, basketball, boxing, judo, disabled track and field, badminton, hockey, golf, martial arts, bodybuilding, karate, football and futsal were selected as study samples and asked to complete the nutritional knowledge and behavior inventory. Referring to the Department of Youth and Sports, the above-mentioned athletes were recruited for the study. The samples included 247 (164 
male and 83 female) athletes. The procedure was explained to the participants. Once the verbal consent was taken, questionnaires were distributed among athletes. The standard nutritional knowledge inventory developed by Zawila et al. [1] was used for the study. Arazi and Hosseini [8] reported its reliability at 0.81 by using Cronbach's alpha. Nutritional behavior was tested by a questionnaire developed by Karegarfard et al. [9], of which reliability was estimated at 0.86 using Cronbach's alpha. The nutritional knowledge inventory included 63 questions in two categories: (1) nutritional knowledge of athlete (19 questions); and (2) general nutritional knowledge (44 questions) in 12 subscales including carbohydrates (5 questions), proteins (3 questions), fats (4 questions ), calcium (4 questions), iron (6 questions), vitamins (9 questions), fiber (2 questions), vegetables (4 questions), water (5 questions), caffeine (2 questions), nutrient and metabolism (4 questions), weight loss (4 questions), and nutritional attitude (11 questions). The nutritional behavior inventory included 11 questions. BMI (body mass index) was calculated by dividing the weight, measured with a digital scale in $\mathrm{kg}$, by the square height in meter, measured with a stadiometer (Seca 217. Stable stadiometer, $0.1 \mathrm{~mm}$ accuracy, Germany). Data were analyzed by the SPSS v.19 software. One-way ANOVA was used to evaluate between groups differences, and Pearson correlation coefficient was used to examine the possible relationship between variables; a $p$ value of $<0.05$ was selected as the level of significance. Given that no particular sampling was required, the ethics committee review was not necessary. However, the procedure was explained to participants and their verbal consent was taken beforehand.

\section{Results}

Most of the participants were male (66.4\%) and the rest were female. The average age was 23.26 for male athletes and 22.59 for female athletes. Moreover, mean BMI was higher for men than women (24.1 \pm 5.3 for men and $21.69 \pm 3.4$ for women) (Table 1).

The findings showed a significant difference in nutritional knowledge of athletes based on gender and education levels. The female athletes scored higher in nutritional knowledge than male athletes; moreover, nutrition knowledge was significantly higher in athletes with a first level of academic education in comparison to participants with High school Diploma and lower degrees. However, no significant difference was found in nutritional knowledge of athletes for age, neither significant difference was found in nutritional knowledge of athletes with higher education (34.92 \pm 9.25) in comparison to the other two groups (Table 2).

In contrast to higher nutritional knowledge of female athletes than male athletes, male athletes scored significantly higher for nutritional behavior than female athletes (55.70 \pm 12.81 and $51.64 \pm 12.92$, respectively); however, both groups had average scores. Additionally there were no significant differences on athlete's nutritional behavior regarding to age and education levels variations (Table 3).

The findings also indicated a significant difference in nutritional knowledge between athletes of different fields of sport. The follow-up Tukey test showed a significant difference in nutritional knowledge of futsal players and handball players $(p=0.016)$ and karate players and handball players $(p=0.035)$.

Table 1 Demographics characteristics of athletes in Khorramabad, 2013.

\begin{tabular}{lll}
\hline \multirow{2}{*}{ Variable } & \multicolumn{1}{c}{ Athletes $(n=247)$} \\
\cline { 2 - 3 } & Males & Females \\
\hline Number $(\%)$ & $164(66.4)$ & $83(33.6)$ \\
Age (years) & $23.26 \pm 4.8$ & $22.59 \pm 5.3$ \\
Height $(\mathrm{cm})$ & $178.83 \pm 9.5$ & $164.25 \pm 5.8$ \\
Weight $(\mathrm{kg})$ & $76.85 \pm 14.3$ & $58.58 \pm 10$ \\
BMI $\left(\mathrm{kg} / \mathrm{m}^{2}\right)$ & $24.1 \pm 5.3$ & $21.69 \pm 3.4$ \\
\hline
\end{tabular}


Table 2 Mean and standard deviation of nutritional knowledge of professional athletes in Khorramabad, 2013 in terms of demographic variables.

\begin{tabular}{|c|c|c|c|c|c|}
\hline & & Number (\%) & $\begin{array}{l}\text { Mean } \pm \text { SD (out of } \\
60 \text { ) }\end{array}$ & Percent (out of 100) & $\dagger$ value \\
\hline \multirow{3}{*}{ Gender } & Male & $164(66.4)$ & $31.87 \pm 7.21$ & 50.58 & \multirow{3}{*}{$-2.11^{*}$} \\
\hline & Female & $83(33.6)$ & $33.88 \pm 7.03$ & 53.78 & \\
\hline & $<19$ & $43(17.4)$ & $31.41 \pm 4.82$ & 49.85 & \\
\hline \multirow[t]{3}{*}{ Age (year) } & $19-29$ & 172 (69.6) & $32.41 \pm 7.22$ & 51.44 & \multirow[t]{3}{*}{2.22} \\
\hline & $>29$ & $32(13)$ & $34.84 \pm 9.18$ & 55.30 & \\
\hline & Diploma and lower & $122(49.4)$ & $31.34 \pm 6.81$ & 49.75 & \\
\hline \multirow[t]{2}{*}{ Education } & Associate and bachelor & $112(45.3)$ & $33.57 \pm 7.17$ & 53.29 & \multirow[t]{2}{*}{$3.62^{*}$} \\
\hline & Master and Ph.D. & $13(5.3)$ & $34.92 \pm 9.25$ & 55.43 & \\
\hline
\end{tabular}

Scores are reported as mean \pm standard deviation out of $63 .{ }^{*}$ Significant difference in $p<0.05 . \dagger$ represents -value for comparison of two groups and $f$-value for further comparison of two groups.

Table 2 Nutritional behavior of professional athletes in Khorramabad, 2013 in terms of demographic variables.

\begin{tabular}{lllll}
\hline \multirow{3}{*}{ Gender } & Male & Number (\%) & Mean \pm SD (out of 100) & $\dagger$ value \\
& Female & $164(66.4)$ & $55.70 \pm 12.81$ & $2.34^{*}$ \\
\multirow{3}{*}{ Age (year) } & $<19$ & $83(33.6)$ & $51.64 \pm 12.92$ & 0.25 \\
& $19-29$ & $43(17.4)$ & $53.06 \pm 11.42$ & $54.56 \pm 13.21$ \\
\multirow{3}{*}{ Education } & $>29$ & $172(69.6)$ & $54.87 \pm 13.91$ & 1.85 \\
& Diploma and lower & $32(13)$ & $52.74 \pm 13.02$ & $55.82 \pm 12.35$ \\
\hline
\end{tabular}

Generally, futsal players had a higher nutritional knowledge than other athletes $(38.5 \pm 2.37)$ and handball players had the lowest level of nutritional knowledge (27.2 \pm 6.07) (Table 5).

The follow-up Tukey test showed a significant difference in nutritional knowledge of judo and taekwondo players (35.43 vs. 25.25, $p=0.038$ ) and bodybuilding and taekwondo players (37.37 vs. 25.25 , $p=0.048)$.

The results of Pearson correlation test demonstrated no significant relationship between nutritional behavior and knowledge of athletes ( $r=0.09$, Table 6 ).

Also, $65.2 \%$ of participants negatively responded to the question that whether they followed a special diet. This indicates that the studied athletes did not follow a special diet. Most of these athletes set up their own diet, and the coaches had no particular role in providing their diet. Moreover, most of the athletes (87.4\%) had three or more than three meals a day and most of them (64\%) used supplements to improve their athletic performance. Also, $69.6 \%$ of the athletes participated in nutritional classes.

According to Table 5, the most important sources of nutritional information were coaches, textbooks, nutrition courses, nutrition consultants, and magazines and newspapers, respectively.

\section{Discussion}

The main findings of the present study signify education levels are dependent on difference of nutrition knowledge and gender related differences in nutrition knowledge and nutritional behavior of male and female athletes. In addition, the results revealed a significant difference in nutritional knowledge and nutritional behavior of athletes in different fields of sport $(p<0.005)$.

The results indicated a difference in nutritional knowledge and behavior of male and female athletes. Female athletes scored higher in nutritional knowledge and lower in nutritional behavior. In most previous studies on nutritional knowledge and habits of male and female athletes, female athletes had higher 
Table 4 The comparison of nutritional knowledge of professional athletes in various fields of sport in Khorramabad, 2013.

\begin{tabular}{|c|c|c|c|c|}
\hline Fields of sport & $\mathrm{N}$ & Mean \pm SD & Percent (out of 100) & Sig. \\
\hline Boxing & 7 & $27.86 \pm 7.58$ & 44.22 & \multirow{24}{*}{$0.005^{* *}$} \\
\hline Freestyle wrestling & 10 & $32.4 \pm 8.58$ & 51.43 & \\
\hline Greco Roman wrestling & 11 & $31.1 \pm 7.22$ & 49.37 & \\
\hline Volleyball & 9 & $32.67 \pm 6.3$ & 51.86 & \\
\hline Track and field & 16 & $33.56 \pm 6.48$ & 53.27 & \\
\hline Badminton & 3 & $29.67 \pm 6.66$ & 47.11 & \\
\hline Basketball & 2 & $28.5 \pm 3.53$ & 45.24 & \\
\hline Futsal & 10 & $38.5 \pm 2.37$ & 61.11 & \\
\hline Judo & 14 & $35.43 \pm 7.77$ & 56.24 & \\
\hline Golf & 16 & $34.5 \pm 7.14$ & 54.76 & \\
\hline Disabled track and field & 11 & $31.55 \pm 7.6$ & 50.11 & \\
\hline Martial arts & 39 & $33.2 \pm 2.37$ & 52.71 & \\
\hline Karate & 18 & $36.33 \pm 6.13$ & 57.66 & \\
\hline Soccer & 11 & $29.73 \pm 6.65$ & 47.19 & \\
\hline Bodybuilding & 8 & $37.37 \pm 5.47$ & 59.32 & \\
\hline Chess & 3 & $30.66 \pm 2.08$ & 48.66 & \\
\hline Handball & 15 & $37.2 \pm 6.07$ & 43.17 & \\
\hline Shooting & 5 & $29.2 \pm 11.4$ & 46.35 & \\
\hline Weightlifting & 7 & $29.28 \pm 4.23$ & 46.47 & \\
\hline Ancient sport & 6 & $33.5 \pm 6.06$ & 53.17 & \\
\hline Kabaddi & 7 & $30.43 \pm 4.3$ & 48.30 & \\
\hline Table tennis & 3 & $34.3 \pm 3.2$ & 54.44 & \\
\hline Taekwondo & 4 & $25.25 \pm 4.92$ & 40.07 & \\
\hline Hockey & 10 & $30.5 \pm 6.98$ & 48.41 & \\
\hline
\end{tabular}

Scores are represented as mean \pm standard deviation and percent out of 100 . $*$ Significant difference $p<0.05$.

Table 5 The sources of athlete's nutritional information.

\begin{tabular}{lll}
\hline \multirow{2}{*}{ Sources of information } & Athletes & \\
\cline { 2 - 3 } & Number & Percent \\
\hline Coach & 79 & 32 \\
Nutrition consultant & 29 & 11.8 \\
Magazines and newspapers & 22 & 8.9 \\
Textbooks & 45 & 18.2 \\
Nutrition courses & 36 & 14.6 \\
Other responses & 36 & 14.5 \\
Total & 247 & 100 \\
\hline
\end{tabular}

nutritional knowledge than male athletes [11, 12], while in contrast Georgia et al. [13] found that nutritional knowledge was higher in men. Karegarfard and Sadeghi [9], by examining 190 collegiate track and field athletes (110 male and 80 female) participating in National Championships in 2005, found no significant difference between male and female students in spite of higher scores of males in nutritional knowledge. In general, they asserted that nutritional knowledge of both male and female Iranian student athletes is low.

Different levels of sports and competitions can influence nutritional knowledge and behaviors of athletes. Previous history of participation in national championship league, national team camps, world championships and the Olympics games which are held under the supervision of a registered dietitian are effective factors to be addressed in future works [10]. 
The follow-up study conducted by Jacobson et al. [15] to compare nutritional knowledge and behaviors of American athletes showed that women received more nutritional information than men $(60.6 \%$ versus 49.5\%). Maughan et al. [16] reported that nutritional behavior of women was better than men; they found that female athletes tended to use less energy intake than male athletes participating in sporting events, even if the difference related to body weight and lean body mass matched, while female athletes tolerated the same training load as male athletes. These investigators also asserted that the energy intake of female athletes was only sufficient to meet their energy and daily works.

According to our results higher educational level is associated with higher nutritional knowledge of athletes; although, only there were significant differences between nutritional knowledge of high school diploma or lower and the athletes with a bachelor's degree or associate degree. Low frequency of athletes with a master's degree and $\mathrm{PhD}$ (about 5\%) can be associated with insignificant difference in nutritional knowledge and behavior of this group and the groups with lower degrees; however, other factors such as gender, history of participation in training courses and participation in championships cannot be ignored in this regard. Unlike most countries, Iranian students do not acquire nutrition education as part of school curriculum; this leads to limited information after finishing school and high school [3]. Therefore, lower nutritional knowledge of athletes with high school diploma or lower compared to athletes who have completed university degrees can be attributed to different training courses.

Rezaeei and Kordestani [14] by evaluating nutritional knowledge, attitude and behavior of elementary and middle school students of Lorestan Province $(N=2,312)$ in 2010 , they reported that $19.9 \%$ of students had good knowledge, 31.5\% had good attitude and $14.8 \%$ had good nutritional behavior. Furthermore, middle school students had better nutritional knowledge and attitude than primary school students, while primary school students had better nutritional behavior. This shows that older students have declining nutritional behavior scores despite their increasing nutritional knowledge and attitude. Their results showed that a small percentage of adolescents had optimal nutritional performance and, in many cases, their nutritional knowledge was not consistent with their attitude and performance.

Our results showed a significant difference in nutritional knowledge of athletes in different fields. The present study compared nutritional knowledge of athletes in 24 fields. The total mean score obtained for nutritional knowledge by athletes was $51.65 \%$ of maximum possible score.

Walsh et al. [17] by assessing nutritional knowledge and behavior of 203 rugby players aged 15-18 years, showed that fat percentage of athletes ranged from 5.1 to $25.3 \%$; moreover, $68 \%$ had normal body fat (10-20\%) and 32 subjects (22\%) were classified as underweight ( $<10 \%$ body fat) and 9.7\% ( $n=14$ ) were classified as obese. In present study $17 \%$ ere categorized as overweight and 5.7\% considered as obese (data were not shown). Walsh et al. [17] reported that the majority of athletes had normal body fat; despite positive attitude to nutrition, most athletes had poor nutritional knowledge and behavior. In addition, nutritional knowledge and behavior of young athletes could be promoted through nutritional education.

Cupisti et al. [18] studied nutritional knowledge and diet of 60 athlete and 59 non-athlete adolescents (14-18 years old) in Italy. They showed that daily energy consumed by female athletes and non-athletes was equal and lower than the recommended level in both groups. Athletes gained more energy from breakfast than non-athletes (18.5 $\pm 6.6 \%$ versus $15 \pm$ $8.2 \%$ ), while the present study reported that 55 of the athletes (22.3\%) eliminated breakfast from their diet. Moreover, athletes had slightly more correct responses to the nutritional knowledge inventory than 
non-athletes (77.6\% versus $71.6 \%)$. This is largely different from correct responses of athletes to the current nutritional knowledge inventory (51.65\%). In total, results of nutritional knowledge and dietary recall inventory represent some incorrect perceptions and nutritional deficiencies; however, the results were better for athletes than non-athletes, which may suggest a role of exercise on nutritional knowledge and behavior.

Jessri et al. [3] by assessing nutritional knowledge of collegiate soccer players and basketball players of medical universities and non-medical universities of Tehran, showed that nutritional knowledge of collegiate students of medical universities and people who acquired nutritional information from reliable sources was higher. These researchers indicated that nutritional knowledge of athletes was improper. They noted that non-standard level of nutritional knowledge could contribute to poor nutritional behavior. This study suggests that athletes can make effective use of education and training related to nutrition.

Through a follow-up study, Jacobson et al. [15] compared nutritional information, knowledge and behavior of collegiate teams of 16 American universities during 1992 and 2001. Consistent to the present study, women (60.6\%) acquired more nutritional information than men (49.5\%). The main sources of nutritional information were fitness trainers for men, while college classes and nutritionists were the first sources of nutritional information for women. In the present study, the predominant sources of nutritional sources included coaches, nutrition textbooks, nutrition courses, and nutrition consultant, respectively. Jacobson and associate reported small changes in nutrition during 6 years' time periods despite previous recommendations on nutritional education to high-level athletes. To our knowledge, this is the first study on nutritional knowledge and behavior of athletes in Khorramabad; therefore, follow-up studies of this kind can be considered as a basis for evaluation of developing nutritional programs in different periods. Evaluating nutritional knowledge and recommendations of 55 trainers, Juzwiak et al. [19] observed that Brazilian trainers responded correctly to $70 \%$ of nutritional knowledge inventory. Nevertheless, no difference was found in nutritional knowledge of trainers of different sports (gymnastics, judo, swimming and tennis) ( $p=0.61$ ).

Consistent to the present study, P. D. Douglas and J. G. Douglas [12] showed that young female athletes had better nutritional knowledge, while weaker nutritional pattern compared to men. Moreover, a significant relationship was found between the type of sport, championship history and nutritional knowledge. High school athletes stated that their parents are the best source of nutritional knowledge. The mean of correct responses was $26.4 \%$ of 48 responses to nutritional knowledge inventory. Jazayeri and Amani [20] studied nutritional behaviors of 31 soccer players with a mean age of 18 years. They reported that athletes had 3.6 meals a day and 4.8 meals a week. A high percentage of athletes consumed pre-cooked foods (55\%) and dietary supplements (42\%) and commonly creatine (36\%). Although $90 \%$ of the athletes knew the importance of hydration status, more than $50 \%$ believed that protein is essential for muscle growth and development as a primary source of muscle energy and mineral supplements and vitamins can raise energy levels. These findings suggest that the athletes required education about healthy dietary practices and nutritional supplements. This not only helps to improve performance, but also causes a healthy dietary practice over time. One of the most important problems of athletes is their wrong nutritional attitudes and behaviors, consumption of food supplements and unbalanced food intake [9].

Our findings revealed no correlation between nutritional knowledge and dietary behavior of athletes. Heanys et al. [21] reported a weak positive correlation $(r<0.44)$ between nutritional knowledge and food intake pattern in 5-9 studies reviewed. In a study of 101 swimmers (13-20 years) in Canada, half of the 
athletes believed that vitamin B supplements provide great energy and drinking water during exercise reduces athletic performance [22]. The correlation between nutritional knowledge, attitude and behaviors can shed light on the fact that increased knowledge influences nutritional attitudes and behavior [23]. Training can be in the form of seminars, lectures, workshops, group discussions, specialized consulting, published magazines, manuals, newsletters and pamphlets.

Nutritional information is delivered through various sources such as coaches, family, teammates, trainers, fitness trainers, parents, magazines and textbooks, suppliers of supplement, Internet and video media. The present study's results showed that athletes (32\%) know their coaches as the first source of nutritional information. In some previous studies, athletes considered their coaches as the first priority for acquiring nutritional information [3, 17], while Karegarfard and Sadeghi [9] found that nutrition textbooks were the first source of information for subjects. In a study conducted by Jessri et al. [3] $89.4 \%$ of athletes reported the trainers as the main source of nutritional information, while Walsh et al. [17], claimed that information sources included trainers (66.9\%), magazines (42.1\%), internet (38.8\%), the athletes themselves (35.5\%), family (28.1\%), sports organizations (16.5\%) and healthcare providers (8.2\%). The main reasons of athletes to consult with their trainers could be easy access, regular communication, athlete-trainer trust and age requirements of young athletes. In other studies conducted on students, parents are described as the first source of nutritional information [12].

Assessing nutritional knowledge of collegiate soccer players and basketball players of medical and non-medical universities of Tehran, Jessri et al. [3] showed that collegiate athletes of medical universities and people who acquired nutritional information from reliable sources had higher nutritional knowledge. Chapman and assistant evaluated the effect of training courses on nutritional knowledge of 72 women from 8 collegiate teams and observed a significant difference in nutritional knowledge caused by training courses; however, no improvement was observed in the consumed foods and food choices because of the short period of the study [24].

\section{Conclusion}

The main findings of this study included average or poor nutritional knowledge and behavior of athletes, gender-based and education-based differences in nutritional knowledge of athletes and differences in nutritional behavior of male and female athletes. Given the vital importance of proper nutritional knowledge and behaviors for successful athletic performance and healthy life style, it is recommended to provide nutritional training courses, seminars, textbooks, nutrition bulletins and pamphlets for each sport-specific, publish nutritional behaviors of international and national elite athletes, manage financial and human resources (using nutrition experts and consultants and promoting nutritional knowledge of trainers), provide workshops and training sessions to enhance nutritional knowledge and behaviors of young athletes in coordinate work of Department of Youth and Sports and scholars is required.

\section{Acknowledgement}

The authors appreciate moral and financial support of the Vice Chancellor for Research and Technology Department, Lorestan University of Medical Sciences and all the athletes for their assistance.

\section{References}

[1] Zawila, L. G., Steib, C. S., and Hoogenboom, B. 2003. "The Female Collegiate Cross-Country Runner: Nutritional Knowledge and Attitudes.” Journal of Athletic Training 38 (1): 67.

[2] Rosenbloom, C. A., Jonnalagadda, S. S., and Skinner, R. 2002. "Nutrition Knowledge of Collegiate Athletes in a Division I National Collegiate Athletic Association Institution.” Journal of the American Dietetic Association 102 (3): 418-20. 
[3] Jessri, M., Jessri, M., RashidKhani, B., and Zinn, C. 2010. "Evaluation of Iranian College Athletes' Sport Nutrition Knowledge.” International Journal of Sport Nutrition 20 (3): 257.

[4] Lin, W., and Lee, Y. W. 2005. "Nutrition Knowledge, Attitudes, and Dietary Restriction Behavior of the Taiwanese Elderly." Asia Pac J ClinNutr 14 (3): 221-9.

[5] Azemati, B., Heshmat, R., Sanaei, M., Salehi, F., Sadeghi, F., Ghaderpanahi, M. et al. 2013. "Nutritional Knowledge, Attitude and Practice of Iranian Households and Primary Health Care Staff: Nutrikap Survey.” Journal of Diabetes \& Metabolic Disorders 12 (1): 12.

[6] Wardle, J., Parmenter, K., and Waller, J. 2000. "Nutrition Knowledge and Food Intake.” Appetite 34 (3): 269-75.

[7] Beals, K. A., and Manore, M. M. 1998. "Nutritional Status of Female Athletes with Subclinical Eating Disorders.” Journal of the American Dietetic Association 98: 419-25.

[8] Arazi, H., and Hosseini, R. 2012. "A Comparison of Nutritional Knowledge and Food Habits of Collegiate and Non-collegiate Athletes.” Sport Logia 8 (2): 100-7.

[9] Karegarfard, M., and Sadeghi, H. R. 1385. "Nutrition Knowledge and Habits of Track and Field Student Athlete of Iran. Research in Biological Science.” 4 (1): 81-96 (in Persian).

[10] http://www.who.int.Global Database on Body Mass Index, BMI Classification. Last update: 15/09/2014.

[11] Azizi, M., Rahmani-Nia, F., Malaee, M., Malaee, M., and Khosravi, N. 2010. "A Study of Nutrition Knowledge and Attitudes of Elite College Athletes in Iran.” Brazilian Journal of Biomotricity 4 (2): 105-12.

[12] Douglas, P. D., and Douglas, J. G. 1984. "Nutrition Knowledge and Food Practices of High School Athletes.” The Journal of the American Dietetic Association 84 (11): 1261-3.

[13] Georgia, S., Guldan, Y. W., Lin, Y., Zhao, M., Xiang, D. P., Yang, L., and Long, F. 1993. "Evaluation of a Nutrition Education Activity for Medical Student in China.” Asia Pacific J ClinNutr 2: 71-6.

[14] Rezaeei Nadia, K. F. 2010. “The Investigation of Lorestan Provience Elementary Student's Nutrition, Attitude, and Behavior in 2010.” [original research (Lorestan Education Research Center, Research Abstract
Book 2010 (in Persian)].

[15] Jacobson, B. H., Sobonya, C., and Ransone, J. 2001. "Nutrition Practices and Knowledge of College Varsity Athletes: A Follow-up." The Journal of Strength \& Conditioning Research 15 (1): 63-8.

[16] Maughan, R. J., and Soltani, V. 2002. “A Comprehensive Guideto Sports Nutrition.” Kharesganazad University of Esfahan, Iran.

[17] Walsh, C. O., Ziniel, S. I., Delichatsios, H. K., and Ludwig, D. S. 2011. "Nutrition Attitudes and Knowledge in Medical Students after Completion of an Integrated Nutrition Curriculum Compared to a Dedicated Nutrition Curriculum: A Quasi-Experimental Study.” BMC Medical Education 11 (1): 58.

[18] Cupisti, A., D'Alessandro, C., Castrogiovanni, S., Barale, A., and Morelli, E. 2002. "Nutrition Knowledge and Dietary Composition in Italian Adolescent Female Athletes and Non-athletes." International Journal of Sport Nutrition and Exercise Metabolism 12 (2): 207-9.

[19] Juzwiak, C. R., and Ancona-Lopez, F. 2004. "Evaluation of Nutrition Knowledge and Dietary Recommendations by Coaches of Adolescent Brazilian Athletes.” International Journal of Sport Nutrition and Exercise Metabolism 14 (2): 222-35.

[20] Jazayeri, S., and Amani, R. 2004. "Nutritional Knowledge, Attitudes and Practices of Bodybuilding Trainers in Ahwaz, Iran.” Pakistan Journal of Nutrition 3 (4): 228-31.

[21] Heaney, S., O’Connor, H., Michael, S., Gifford, J., and Naughton, G. 2011. "Nutrition Knowledge in Athletes: A Systematic Review.” Int J Sport NutrExercMetab 21 (3): 248-61.

[22] Campbell, M., and MacFadyen, K. 1984. "Nutrition Knowledge, Beliefs and Dietary Practices of Competitive Swimmers.” Canadian Home Economics Journal (Revue canadienned'economiefamiliale).

[23] Hamailei Mehrabanei, H. P., Alaian, F., and Azizei, F. 2009. "Changes in Knowledge, Attitude and Practice of Nutritional District 13 of Tehran after 4 Years of Education.” Journal of Endocrinology and Metabolism 11 (3): 235-45.

[24] Chapman, P., Toma, R. B., Tuveson, R. V., and Jacob, M. 1997. "Nutrition Knowledge among Adolescent High School Female Athletes.” Adolescence 32 (126): 437-46. 